\title{
Author Correction: MX2-mediated innate immunity against HIV-1 is regulated by serine phosphorylation
}

Gilberto Betancor (D), Jose M. Jimenez-Guardeño (D), Steven Lynham, Robin Antrobus, Hataf Khan, Andrew Sobala, Matthew D. J. Dicks and Michael H. Malim (D)

Correction to: Nature Microbiology, https://doi.org/10.1038/s41564-021-00937-5, published online 19 July 2021.

In the version of this article initially published, labels on the top row of gels in Figure 4d originally read "S14D/S17D/S18D"; they have been corrected to read "P-NTD." The error has been corrected in the HTML and PDF versions of the article.

Published online: 13 August 2021

https://doi.org/10.1038/s41564-021-00960-6

(C) The Author(s), under exclusive licence to Springer Nature Limited 2021 